\title{
POLÉMIQUE(S) ET DISCOURS RACISTE
}

\section{Gaetan Clavien $^{1}$}

"Les conflits se jouent aujourd'hui, pour une large part, sur la scène médiatique : ils s'y nouent ou s'y dénouent, même s'ils ne s'y résolvent pas"2. Il en va ainsi de maintes controverses, plus ou moins passionnées et plus ou moins virulentes -que l'on pense par exemple aux polémiques médiatiques dans le cadre de ce qu'il a été convenu d'appeler "l'affaire du foulard"3 en France ou aux polémiques entourant les votations concernant les Étrangers en Suisse ${ }^{4}$, ou encore à l'affaire dite "des fonds juifs et de l'or nazi", qui a mobilisé le débat public en Suisse de 1995 à 1999. Ces polémiques se distinguent toutefois par un trait commun : elles se nouent autour de la question de l'Altérité et, à chaque fois, tout un pan de l'activité polémique liée à ces événements s'est appuyée sur des catégorisations racisantes. C'est cette "zone d'ombre" de la polémique comme lieu occasionnel

Assistant à l'Université de Genève (DEA médias et Département de sociologie).

2 B. DELForCE, "La responsabilité sociale du journaliste: donner du sens", Les Cahiers du journalisme, $\mathrm{n}^{\circ} 2$, décembre 1996, p. 25.

3 P. Siblot, "Ah! Qu'en termes voilés ces choses-là sont mises", Mots, $\mathrm{n}^{\circ} 30$, mars 1992, pp. 5-16.

4 A ce sujet voir notamment M. EBEL et P. FIALA, Sous le consensus, la xénophobie : paroles, arguments, contextes : (1961-1981), Lausanne, Institut de science politique, 1983 et U. WINDISCH, Xénophobie? : Iogique de la pensée populaire : analyse sociologique du discours des partisans et des adversaires des mouvements xénophobes, Lausanne, L'Age d'homme, 1978. 
d'actualisation des stéréotypes et des préjugés racistes que nous chercherons à étudier dans cette communication, afin d'en discuter les enjeux par rapport au champ médiatique.

\section{Un "mode exceptionnel d'interaction discursive"1}

Le propre de la polémique réside dans la nature de l'échange verbal ou textuel (on parle de confrontation ou d'affrontement) -composante "guerrière" que signale son étymologie. Elle est ainsi généralement marquée par ce statut particulier de "petite guerre ou fantasia, simulacre et substitut de la guerre littérale"2. Même si elle se situe, suivant Greive, "en deçà d'une norme de bienséance et d'objectivité" 3 et même si elle peut s'avérer virulente, la polémique reste un rituel particulier et légitime, qui permettrait certes écarts et violences, sans toutefois que les conséquences ne dépassent par trop le cadre de la joute polémique. Dans un contexte médiatique, les protagonistes connaissent le cadre d'interaction dans lequel ils évoluent, ils s'y comportent suivant les règles du genre, que les spectateurs, à leur tour, reconnaissent et admettent. Toutefois, ce statut spécifique qui caractérise la polémique apparaît quelque peu équivoque, comme le souligne Wachsman : "Elle se lit avec plaisir (et s'écrirait bien, avec une allégresse non dissimulée, pour combattre les idées et les individus qui nous agacent), mais ce n'est, tout de même, pas très convenable dans une société qui se veut policée..."4.

Cette ambiguité apparaît très clairement problématique dans le cas de polémiques telles que celle qui a entouré l'affaire des fonds en déshérence ${ }^{5}$ et qui a pris un tour particulièrement virulent en janvier

1 L'expression est de C. PLANTIN, "Des polémistes aux polémiqueurs", in G. DeClerQ et al., La parole polémique, Paris, Champion, 2003, p. 390.

2 C. Kerbrat-Orecchioni, "La polémique et ses définitions", in N. Gelas (eds.), Le discour's polémique, Lyon, Presses Universitaires de Lyon, 1980, p. 6.

3 A. Greive, "Comment fonctionne la polémique", in G. RoEllen BLECK, $L e$ discours polémique : aspects théoriques et interprétations, Tübingen, G. Narr, 1985, p. 20.

4 P. WAChSMAn, "La polémique face au droit de la presse", in G. DECLERQ et al., La parole polémique, Paris, Champion, 2003, p. 297.

5 L'affaire dite des "fonds juifs", ou des "fonds en déshérence" désigne un ensemble complexe et intriqué de controverses autour, premièrement, des fonds en déshérence appartenant à des victimes du nazisme non restitués par des institutions privées suisses, deuxièmement, des transactions entre la Banque nationale suisse et 
1997 dans les médias suisses, à la suite d'une interview' de JeanPascal Delamuraz, alors président de la Confédération helvétique :

"Chantage" et "rançon" : depuis que le conseiller fédéral JeanPascal Delamuraz a prononcé ces deux mots il y a quinze jours pour qualifier certaines exigences financières émises par des organisations juives, la controverse ne connaît plus de trêve ${ }^{2}$.

De fait, les réactions s'enchaînent, entre prises de position des acteurs suisses et internationaux, entre blâmes et soutiens. Nous avons retenu ici une séquence de la polémique telle qu'elle s'est nouée dans les courriers de lecteurs autour des propos de Jean-Pascal Delamuraz (notamment au travers de l'utilisation du "site d'énonciation" aménagé par ce dernier), parce qu'elle est marquée par la résurgence dans l'espace public d'un discours antisémite resté jusque-là diffus et latent ${ }^{3}$. En voici trois exemples, que nous étudierons ci-après :

1. Alors bravo Monsieur Delamuraz, vous n'avez pas baissé votre culotte devant le Congrès juif mondial. (...) Et je pense aussi que les juifs de ce congrès, n'ont de leçon à donner à quiconque, car ce qui se passe en Palestine n'est pas plus joli que les barbaries nazies ${ }^{4}$.

2. (...) ces gens-là pour qui, hélas, tout semble se résumer à une question d'argent ! ${ }^{5}$

3. (...) Les grands patrons de la finance mondiale et des banques n'étaient-ils pas et ne sont-ils toujours des gens proches du milieu juif ? 6

le régime nazi et, troisièmement, du comportement de la Suisse durant la Seconde Guerre mondiale, en rapport notamment avec le refoulement de réfugiés juifs.

1 Une interview du 31 décembre 1996 accordée à deux quotidiens suisses romands, le 24 Heures et la Tribune de Genève. Pour une analyse détaillée de l'interview de J.-P. Delamuraz cf. J. WIDMER, "J.-P. Delamuraz prend position : analyser le pouvoir symbolique dans le texte", in J. WIDMER et C. TERzI (eds), Mémoire collective et pouvoirs symboliques : l'affaire dite des fonds juifs et de l'or nazi dans le discours social en Suisse, 1995-1997, Fribourg, Département Sociologie et média, 1999.

2 Le Courrier, 15/01/1997.

3 G. CLAVIEN et A. OTHENIN-GIRARD, Le discours antisémite dans la presse suisse romande, Travaux et communication du département de science politique, 1998.

4 Lettre de lecteur, Tribune de Genève, 11-12/01/1997.

5 Ibid.

6 Ibid. 


\section{Les circonstances de la polémique}

Il faut encore, avant de passer à une brève analyse du fonctionnement polémique du discours raciste, relever certaines "circonstances" qui marquent ce type de polémiques, car celles-ci renforcent l'ambiguïté que nous soulignions précédemment.

Premièrement, ces polémiques sont "banalisées"1, c'est-à-dire dominées par l'expression d'émotions telles que la colère et l'indignation au détriment d'une véritable compétence polémique. Elles sont ensuite "éclatées" sur la scène médiatique, du fait qu'elles se logent principalement dans ces lieux d'opinions que sont les courriers de lecteurs ${ }^{2}$. Elles se caractérisent encore par une forme d'émergence ${ }^{3}$ spontanée, en ce sens qu'elles ne se préparent pas et ne se convoquent pas : on a parlé "d'avalanche" de lettres de lecteurs dans la presse. Elles deviennent enfin imprévisibles, parce que l'on ne sait jamais à quoi elles aboutiront, sans compter que le "mouvement" polémique peut être violent.

\section{Discours polémique et discours raciste}

Parmi les définissants du discours polémique ${ }^{4}$ (le désaccord entre les protagonistes, la falsification des contenus et sa nature argumentative) nous nous concentrerons ici sur le taux de polémicité, autrement dit sur sa visée perlocutoire, la disqualification de la cible. On connaît le principe de cette rhétorique 5 qui utilise 1'attaque "gratuite" ou "basse", l'allusion perfide, l'insinuation diffamatoire, l'amalgame, etc. Autant de procédés auxquels s'ajoute la catégorisation racisante, dont la mécanique de disqualification spécifique est la suivante : la cible se trouve mise en cause au titre même de son appartenance à un

1 Plantin parle d'une banalisation de la polémique : cf. C. PLANTrN, op. cit.

2 Sur l'importance du courrier des lecteur dans la presse suisse : cf. U. WINDISCH, op.cit.

3 La notion d'émergence est empruntée à $\mathrm{H}$. GIANnini, La "réflexion" quotidienne: vers une archéologie de l'expérience, Aix-en-Provence, Alinea, 1992, pp. 64-65.

$4 \mathrm{M}$. TUTESCU, L'argumentation : introduction à l'étude du discours, Bucurest, Éd. Univ. de Bucarest, 1998, p. 397.

5 C. Kerbrat-OrecChioni, op. cit., p. 32. 
groupe catégorisé par les traits stéréotypiques enregistrés et stabilisés comme ses définissants. Ainsi, la réalisation discursive de la disqualification racisante peut s'opérer soit directement, par la désignation groupale, soit indirectement par la mention (ou l'allusion) des prédicats racistes classiques, le tout fonctionnant comme un "système de références, abstrait de la réalité du groupe qui lui sert d'assise"l :

Dans le premier exemple, la désignation de la cible glisse de "Congrès juif mondial" à "juifs de ce congrès". Comme toute désignation catégorielle, celle-ci "éclate de son contenu connotatif. Lorsque je prononce juif ou nègre (noir), femme ou ouvrier, en même temps que je désigne le réel, je tire sur le fil de l'imaginaire que le majoritaire a enroulé sur le réel"'2. Ainsi, être désigné par une catégorie équivaut à être totalement déterminé par celle-ci, c'est-à-dire privé de caractères "propres" en dehors de ceux qui définissent cette catégorie. En l'occurrence, la désignation catégorielle est renforcée par une stratégie d'inversion victime-bourreau.

Dans le second exemple, la désignation "ces gens-là" reste certes anonyme, mais la catégorie "juif" est convoquée par la relative "pour qui, hélas, tout semble se résumer à une question d'argent", qui renvoie au registre classique de la stéréotypie antisémite que sont les finances juives.

Il est cependant clair que l'énonciation des catégories racisées s'est voilée, et ceci particulièrement dans les contextes publics ou semi-publics. Des stratégies plus ou moins conscientes de définition de groupe (l'anonymisation, les constructions impersonnelles, etc.), de légitimation (les arguments d'autorité, etc.) et de dissimulation (l'utilisation du conditionnel, les tournures indirectes, les questions rhétoriques, etc.) seront alors investies dans le discours ${ }^{3}$. Ainsi, le troisième exemple suggère le stéréotype au travers de la question rhétorique. On passe ainsi, comme le souligne Taguieff, "du sens aux effets de sens, de la signification littérale aux connotations"4.

1 C. Gulllaumin, L'idéologie raciste : genèse et langage actuel, Paris, Mouton, 1972 , p. 98.

2 Ibid., p. 183.

3 Pour une présentation complète des différentes stratégies, cf. M. REISIGL et $\mathrm{R}$. WODAK, Discourse and discrimination : rhetorics of racism and antisemitism, London, Routledge, 2001, pp. 31-90.

4 P.-A. TaguiefF, Le racisme, Paris, Flammarion, 1997, p. 51. 


\section{L'intérieur et l'extérieur de la polémique}

Nous voulons insister ici sur le fait que, si la polémique peut occasionner, voire favoriser par l'exacerbation de l'un de ses traits, la catégorisation racisante, les conséquences de ce type de disqualification dépassent le cadre limité de la joute polémique. En effet, la marque du racisme étant l'essentialisation -qui se concrétise dans l'assignation close et irréversible de caractéristiques définissant l'essence d'un groupe, toute actualisation discursive du préjugé raciste entraîne immanquablement la reproduction de la même catégorisation infiniment reposée : "Elle prononce " la chose jugée". L'activité de nommer, la première du rapport à l'“autre", est une activité de liquidation"l souligne Guillaumin. Le discours raciste devient alors la constante reproduction d'un procès qui a déjà eu lieu.

C'est en définitive la polémique comme lieu d'actualisation de ces "verdicts" qui doit être problématisée. Pour ce faire, il s'agit premièrement de prendre en compte l'ensemble de l'espace médiatique dans lequel la polémique se tient, et deuxièmement d'exploiter la distinction opérée par Revel ${ }^{2}$ entre la polémique de fond et la polémique de ton, entre un "intérieur" et un "extérieur" de la polémique, pour le dire autrement.

\section{L'espace médiatique de la polémique}

Une polémique peut comporter des moments d'effervescence voire de défoulement, nous l'avons vu au travers des exemples étudiés. Il va cependant de soi que l'espace médiatique de la polémique est beaucoup plus large que le seul courrier des lecteurs évoqué et que la presse écrite ne se résume pas à un lieu d'exposition de la polémique : elle en est aussi et surtout une instance d'articulation et de gestion, qui effectue des choix notamment par le fait de publier ou non les lettres de lecteurs (voire de les regrouper dans des pleines

1 C. Guillaumin, op. cit., p. 184.

2 J.-F. REVEL, Contrecensures : politique, religion, culture de masse, art et critique $d^{\prime}$ 'art, Paris, J.-J. Pauvert, 1966. 
pages thématiques sous la rubrique "courrier spécial fonds juifs"1), qui sélectionne les points de vue des parties en présence, les acteurs concernés pour les interviewer. Les éditoriaux, pour ne prendre que cet exemple, sont des lieux privilégiés de gestion, de mise en visibilité, mais surtout de mise en lisibilité et en intelligibilité des événements publics ${ }^{2}$ : ils les choisissent, les définissent, les qualifient, les expliquent ; ils les problématisent, les élucident et les évaluent. En ce sens, les éditoriaux offrent la possibilité d'un engagement et d'un positionnement de l'instance médiatique, qui, en retour, se trouve impliquée dès le moment où elle décide de traiter telle information dans l'éditorial -quelle que soit la posture retenue, celle de l'engagement ou bien celle de la neutralité.

C'est donc tout un ensemble de productions, de l'article de fond à l'éditorial, en passant par les interviews, les courriers de lecteurs et les chroniques, qui constituent en dernière analyse l'espace de la polémique, chacune d'entre elles étant un "coup" joué participant à la structuration de la polémique.

\section{Une fonction polémique}

La brève analyse des exemples cités permet de relever un autre aspect important pour notre propos, à savoir que les catégorisations investies au cours de la polémique mobilisent un ensemble de représentations et de préjugés sociaux. Or, ce sont ces représentations que les discours médiatiques vont entretenir, déconstruire ou mettre en débat. Autrement dit, elles forment une toile de fond, une matière sur laquelle les productions journalistiques agissent, pour les conforter, les déconstruire ou les dénoncer. Dans cette optique, Fairclough insiste sur ce qu'il appelle le pouvoir de "signifier" des médias, qu'il définit comme le "pouvoir d'influencer les connaissances, les croyances, les valeurs, les relations sociales et les identités sociales" (nous traduisons) ${ }^{3}$. Ce pouvoir de "donner sens" réside entre autres dans la capacité de gestion et d'articulation de la

1 Cf. Tribune de Genève, 11-12/01/1997.

2 Ces concepts sont empruntés à P. Charaudeau, Le discours d'information médiatique : la construction du miroir social, Paris, Nathan, 1997, p. 220.

3 N. Fairclough, Media discourse, London, Arnold, 1997, p. 2: "a signifying power (the power to represent things in particular ways) which is largely matter of how language is used ". 
polémique propre à l'instance médiatique. C'est à ce niveau que se situent les conditions de possibilité d'une polémique de fond.

Livrée à elle-même, la polémique peut s'emballer et se révéler infamante, comme dans les cas que nous avons évoqués. Elle suppose alors certaines " régulations", qui passent par la mise en intelligibilité des événements, par l'articulation des enjeux qui y sont associés, par une attention particulière apportée à la désignation et à la qualification des acteurs, par la nécessité d'évaluer et de juger, par l'opposition et la dénonciation, par ce " ressaisissement critique " dont parlait Paillet.

Elle implique aussi une posture journalistique spécifique : "Le polémiste de jadis exécutait des embuscades à un point donné de la route où il savait que passerait un convoi de prisonniers à délivrer ; celui d'aujourd'hui doit se débattre au sein d'un océan de boue qui se referme sans arrêt au-dessus de sa tête"1.

Ainsi, dès lors que la dimension "normative" de la polémique comme "mode de traitement privilégié de quelques grands problèmes permanents" 2 est récupérée, l'espace médiatique peut être régulé par l'entrecroisement des voix participant à la dynamique de la polémique.

1 J.-F. REVEL, op. cit., p. 137.

2 La formule est de C. Plantin, op. cit., p. 390. 